\title{
Las ruinas de una company town salinera en la península de Baja California: Isla El Carmen en el Mar de Cortés 1880
}

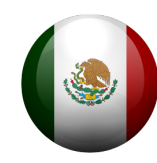

\section{Enrique Esteban Gómez Cavazos}

Arquitecto. Master en Investigación en Urbanismo. Estudiante de doctorado en Urbanismo, Programa de Doctorado de la Universitat Politécnica de Catalunya [Barcelona, España]. Ensenada [Baja California]

México <eecavazos@hotmail.com>.

\section{Resumen}

Este artículo pretende acercarnos a identificar el desarrollo de una colonia industrial dedicada a la extracción y exportación de sal, la cual ayuda al desarrollo económico de una región aislada del centro de México a finales del siglo XIX y durante el siglo XX, a través de inversiones extranjeras. Nos interesa estudiar el alcance urbano del proyecto de las compañías extranjeras y la manera en que se diseñan en el territorio estas poblaciones para verificar la existencia de un patrimonio industrial que pudiera ser reconocido y puesto en valor actualmente.

\section{Palabras clave}

Company town. Salinas. Patrimonio industrial.

\section{The ruins of a salt company town in the peninsula of Baja California: Isla El Carmen in the Sea of Cortés 1880}

\begin{abstract}
This article tries to identify the development of an industrial colony dedicated to the extraction and export of salt, which helps to achieve the economic development of an isolated region of Mexico in the late nineteenth century and during the twentieth century through foreign investments. We are interested in studying the urban scope of the project of foreign companies and the way in which these populations are designed in the territory to verify the existence of industrial heritage that could be recognized and valued at present.
\end{abstract}

\section{Keywords}

Company town. Salt extractions. Industrial heritage. 


\section{Introducción: ubicación y localización de la Isla El Carmen Baja California Sur}

La Isla El Carmen está ubicada en el Mar de Cortés frente al municipio de Loreto en Baja California Sur, es parte de una geografía realmente excepcional con paisajes de desierto, un mar tranquilo y rodeado de montañas secas. La forma de la Isla El Carmen es alargada y el sitio que ocupa es de unas 15,000 hectáreas aproximadamente; tiene unos 28 kilómetros de largo y una cordillera que la recorre con elevaciones de hasta 479 metros sobre el nivel del mar para después hundirse en el mar y volver a levantarse en las costas peninsulares. Desde la ciudad de Loreto en la costa se encuentra a unos 15 kilómetros en barco y actualmente es parte del parque nacional Bahía de Loreto protegido por su rica diversidad natural1.
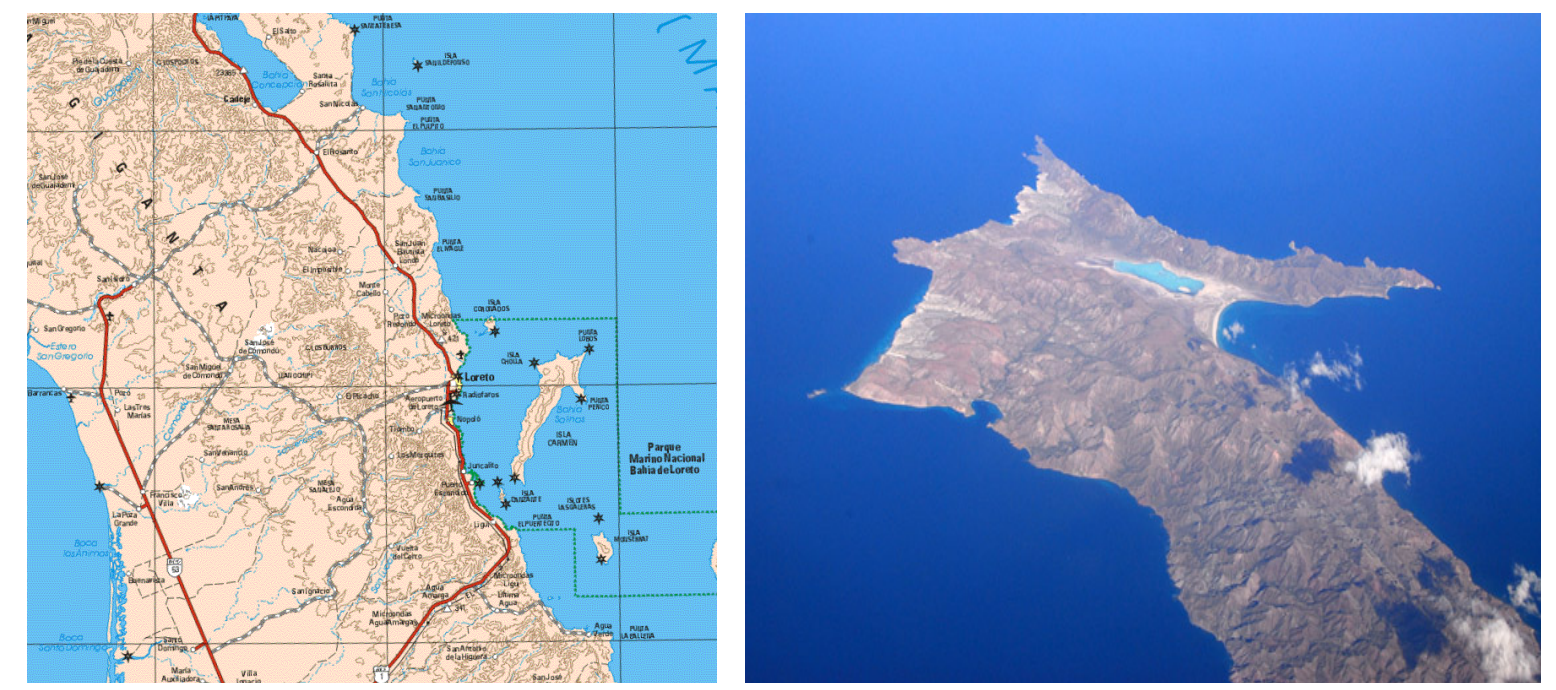

Figura 1. Mapa de ubicación y fotografía aérea de la localización de la isla El Carmen en el Golfo de California. Fuente: www.maps-of-mexico.com

\section{Contexto histórico}

La extracción de sal fue crucial para el crecimiento económico de Baja California Sur. La abundancia y la calidad de los depósitos de sal de la Isla El Carmen fueron reconocidas por los misioneros jesuitas desde el siglo XVII. Algunos de ellos nombraron a la salina de la isla El Carmen inagotable y una de las mejores reservas naturales de sal del mundo.

En 1717 el Padre Juan María Salvatierra hizo una petición al rey de España para explotar la salina, diciendo "hay suficiente sal para abastecer el mundo entero". Edward H. Cook, un ingeniero de minas de Tucson Arizona visitó los depósitos de sal de la Isla El Carmen, reportando que por varios años el gobierno mexicano mantuvo una colonia penal en la isla y trabajaba los depósitos con convictos, pero que con la intervención francesa que sufrió el país la isla fue vendida a capital privado (Busto, 2015).

La historia de la Isla El Carmen está muy ligada al comercio de sal, por sus ricos depósitos localizados en el interior de la isla. Durante el invierno de 1841-1842 el barco Naslednik Aleksandr arriba desde Sitka, Alaska, para recolectar sal para preservar pieles y pescado. Abordo venia I.G. Voznesenskii miembro del Museo Zoológico de la Academia Rusa de Ciencias en San Petersburgo, el hizo una extensa colección botánica en el área del Golfo y probablemente fue el primer europeo en visitar una isla del Golfo de California y recolectar especímenes con fines científicos. Desafortunadamente su colección termina en almacenamiento y no fue hasta un siglo después que fueron descubiertos.

\footnotetext{
1 El parque nacional Bahía de Loreto es un área natural protegida de México, con el carácter de Parque Nacional, cuyo espacio designado como tal se encuentra conformado por la bahía de Loreto, en aguas correspondientes al mar de Cortés y a su vez que son parte del estado de Baja California Sur.
} 
Durante 1850 la demanda de sal incrementa dramáticamente y las reservas de sal de la Isla El Carmen se convierten en la principal fuente para la ciudad de San Francisco California. Para $1867,30,000$ toneladas a 18 dólares la tonelada fueron embarcados, haciendo muy rico al concesionario norteamericano Ben Holladay. Después de estas exitosas exportaciones a mediados del siglo XIX, los dueños de la industria de sal pasa a manos mexicanas y extranjeras.(Aitchison, 2010).

La sal se explota de forma rudimentaria durante casi todo el siglo XIX, pero no es hasta 1870 cuando se explota industrialmente. El cónsul de Estados Unidos en la ciudad de La Paz, Santiago Viosca establece trabajos de extracción de sal en la isla con alrededor de 140 trabajadores, la mina de sal incorpora un ferrocarril de vía angosta y se construyen dos edificios, uno utilizado como oficinas y otro como almacén de maquinaria (Southworth, 1989).

Las expediciones comandadas por Charles H. Townsend en 1911 reportan que la isla El Carmen contiene depósitos de blanca sal como nieve en un lago interior de más de dos millas de largo y una de ancho. La superficie de sal se disuelve durante la temporada de lluvias y después de recristalizarse se crean nuevas reservas que parecen ser inagotables y solo una pequeña parte de la superficie del lago ha sido explotada. Habla de 35,000 toneladas embarcadas por año y que muchas más podrían exportarse si hubiera demanda. La pureza de la sal es tanta que no requiere refinamiento. También menciona que hace cincuenta años que se explota la sal en la isla y que el lago de sal no tiene conexión con el mar (Busto, 2015).
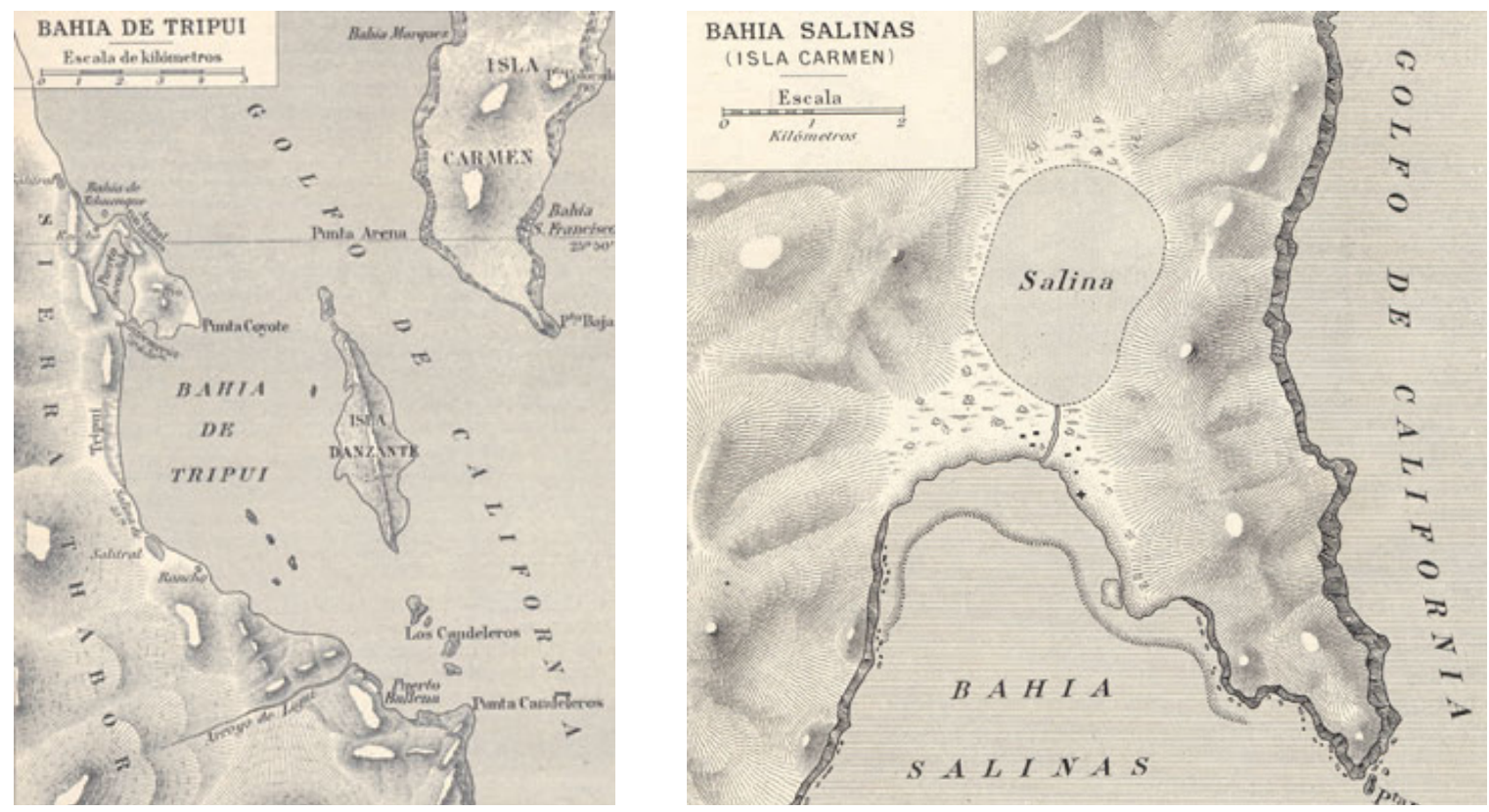

Figura 2. Ubicación de la salina de Isla El Carmen. Imágenes publicadas en el libro Territorio de la Baja California escrito por León Diguet, 1912. Fuente: Historia General de Baja California Sur: La economía regional de Dení Trejo Barajas y Edith Gonzales Cruz.

Figura 3. Dibujo de la Salina de Isla El Carmen. Fuente: The Saltworks on Carmen Island MY JOURNEY TO THE PENINSULA OF BAJA CALIFORNIA. The Journal of San Diego History. SAN DIEGO HISTORICAL SOCIETY QUARTERLY Summer 1977, Volume 23, Number 3. James E. Moss, Editor Thomas L. Scharf, Assistant Editor. By Herman Frederik Carel ten Kate.

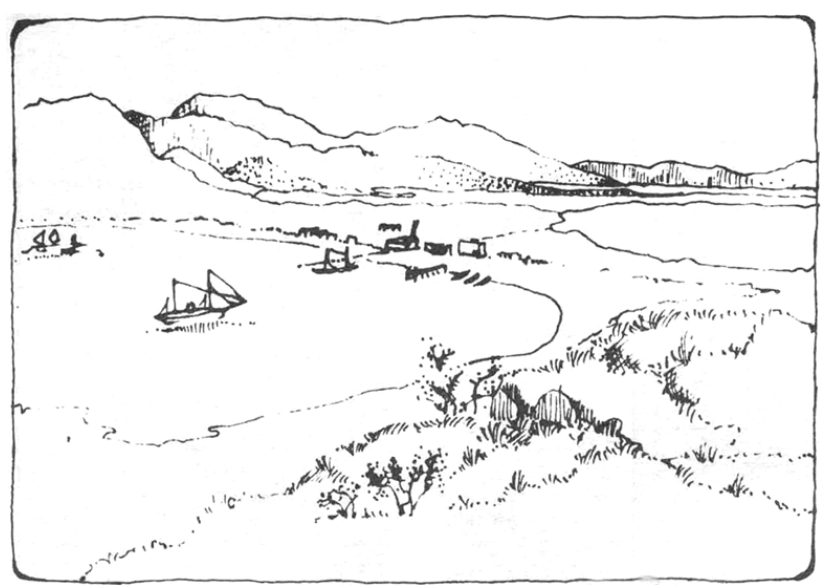


Un producto muy importante, no solo en Baja California sino en todo el mundo es la sal, que se ha utilizado para la conservación de comida, en la minería y en el curado de pieles. Desde tiempos coloniales en México, los depósitos de sal eran considerados propiedad del estado por su gran valor. A pesar del bajo precio del producto las regiones salineras fueron explotadas abundantemente. Sin embargo el aislamiento de la península de Baja California fue una severa limitación para la extracción y transportación de embarques de sal desde los sitios de producción hacia las áreas de consumo en la misma península y el resto de Norteamérica principalmente.

Los comerciantes en Baja California Sur traían harina a Loreto y cargaban quesos, carne, pieles y sal de la isla El Carmen. Los productos derivados del ganado eran tomados de Sonora y Sinaloa, la sal era descargada en el puerto de La Paz de donde se transportaba en carros de bestias a las minas de El Triunfo y San Antonio (centros mineros importantes en el sur de la península). La comercialización de la sal fue hecha principalmente por mexicanos y compañías, ya que el margen de beneficio era menor que el de la minería donde había mucha inversión extranjera. (Ganster, 2007).

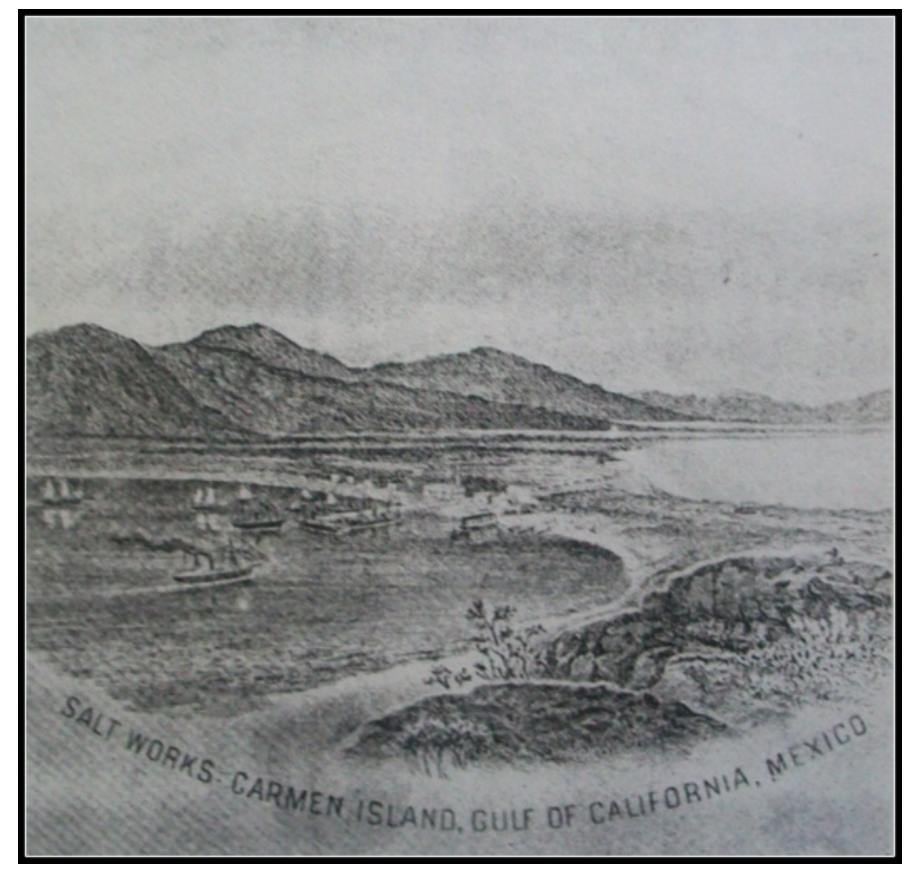

Figura 4. Dibujo que muestra la bahía, la salina y la laguna. Se estima que el dibujo representa los inicios de los trabajos de extracción de sal a mitad del siglo XIX, ya que se alcanzan a ver muy pocas construcciones a pesar de que se aprecian varios barcos. Fuente: Baja California Ilustrado de J. R. Southworth, 1899.

Las políticas económicas del régimen del Porfiriato (1876-1910) restructura el espacio peninsular, la región de Loreto vive una activación económica por medio de la instalación de posiciones políticas del gobierno central que permite la formación de alianzas favorables entre autoridades y empresarios. La explotación de perlas, sal y guano forman un circuito económico que trajo beneficios notables.

El Boom de la explotación de la sal en Baja California Sur surge en 1880 cuando el precio llega a 12 dólares por tonelada. En 1890 el precio se desploma a 3.5 dólares por tonelada a causa de varios factores, como el surgimiento de la industria de sal en otras partes de la costa del pacifico mexicano como Guerrero, Colima, Jalisco, Nayarit, Sinaloa y Sonora. También porque la sal deja de ser utilizada en la minería remplazada por otros químicos.

Hasta 1900 la explotación de la sal era dominada por capital mexicano pero no genera los beneficios esperados como en la minería y la explotación de perlas, aunque genera trabajo y ayuda a fortalecer la red de comercio. En los inicios del siglo XX la Isla El Carmen y su explotación pasan a la Pacific Salt Company Limited of London, compañía que explota las reservas de sal durante el resto del Porfiriato (Ganster, 2007).

Como lo mencionan Erín Castro y Micheline Cariño en su artículo El estudio de los contratos para la explotación de sal durante el Porfiriato en Sudcalifornia: 
[...] se puede apreciar que la explotación de la sal sudcaliforniana fue realizada hasta 1900. La sal, al ser un artículo indispensable para las actividades productivas locales, estuvo dentro de los intereses de los inversionistas locales. Sin embargo, la riqueza salina de Sudcalifornia permitía una explotación a mucho mayor escala, sin afectar al abasto local. Para realizar dicha explotación se requería cierta capacidad naviera de la que carecían los empresarios locales, quienes mostraron mayor interés en utilizar la sal en otras actividades, no tanto en exportarla. Fue así que el gobierno permite que la principal salina sudcaliforniana de la época, pase a manos extranjeras (Castro y Cariño, 2002).

Figura 5. En la fotografía vemos los rieles que existían en la mina de sal de la Isla El Carmen. Estos rieles conectaban la laguna de sal y la población donde se encontraba el muelle frente a Loreto. La estimación de la fotografía es a finales del siglo XIX.

Fuente: vamonosalbable.blogspot.mx/

Figura 6. Fotografía del área donde se colocaban los costales de sal para ser transportados por las vías de ferrocarril hacia los embarques en el muelle.

Fuente: http://www.californax.com/

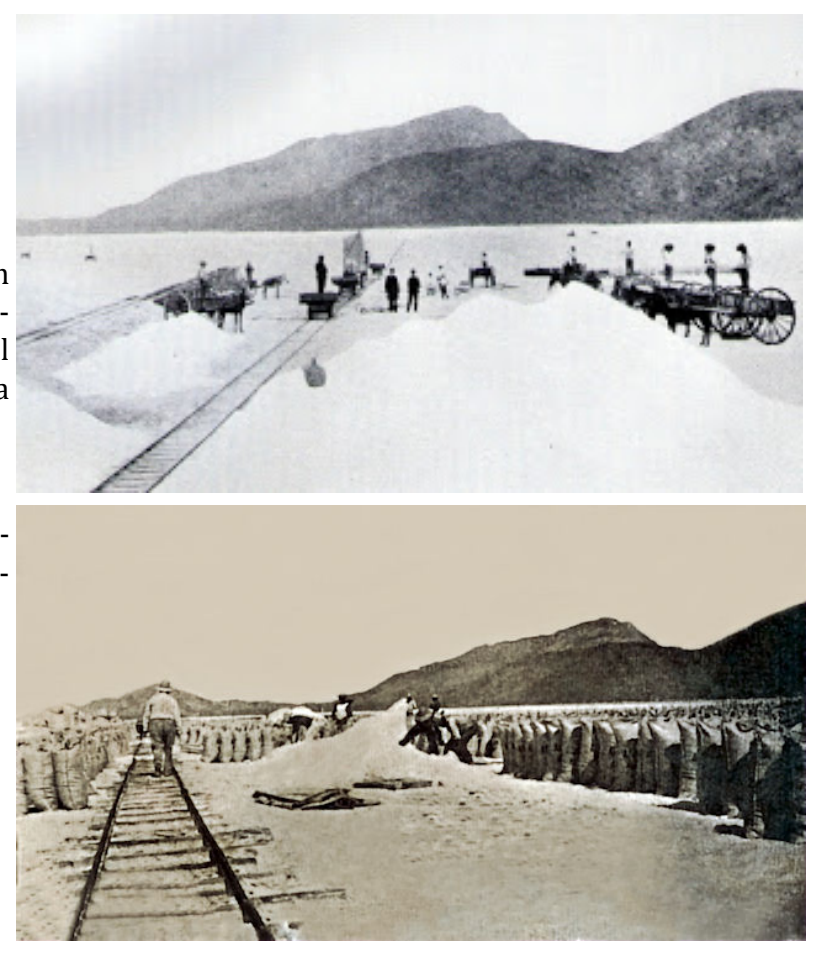

\section{Los Inicios de la company town}

Un dado interesante mencionado es que en 1861 se le entregan a Guillermo Denton doscientas toneladas de sal como pago por la elaboración del plano de la ciudad de La Paz. Se nos hace importante decir que el Ingeniero Guillermo Denton había ejecutado los trabajos de concesiones y mediciones en la península entre 1857 y 1880, este personaje llegado de Inglaterra y de nacionalidad mexicana durante el Porfiriato fue un importante intermediario para las concesiones territoriales en toda la península, el conservaba los planos y documentos originales de muchas obras y exploraciones geográficas mexicanas y estadounidenses. Su nombre aparece en varios mapas y planos de la época donde se reconoce la superficie y la topografía del perímetro peninsular, un deslinde realizado sobre mapas viejos de treinta años (Pacheco y Sánchez, 1997).

En 1867 Fortunato de la Vega traspasa la propiedad de la salina de la Isla El Carmen a la compañía de vapores California, Oregon \& México Steamship Company, una sociedad de capitalistas norteamericanos. Esta compañía extrae una importante cantidad de sal, e instala una maquinaria para beneficiar una mayor cantidad de sal, de la cual una parte era vendida a los buques nacionales que traficaban en las costas peninsulares y otra era enviada a San Francisco en el vapor Continental de su propiedad. Algunas autoridades regionales cuestionaban el traspaso de las concesiones a dominio extranjero ya que no pagaban ningún tipo de impuesto.

A pesar de esta inestabilidad en las concesiones, se percibe una transformación de la explotación salinera como consecuencia de nuevas tecnologías y sistemas de trabajo introducidas por las compañías (Trejo y González, 2002).

Aproximadamente en 1875 cuando los hermanos Viosca de la Paz toman el control de la sal en la isla, como se menciona antes, construyen un ferrocarril de 600 metros donde una locomotora 
movía la sal hacía el pequeño muelle. Eventualmente las reservas de sal cambian de control bajo la compañía Santa Fe Railroad y después a la Pacific Salt Company Limited of London. Con la llegada de inversiones extranjeras los métodos de extracción de sal mejoran y se renueva el equipamiento del ferrocarril, se planea también la población y su estrecha vinculación con la industria salinera. Creemos que después de este periodo la colonia salinera y su estructura urbana funcionan de manera estable.

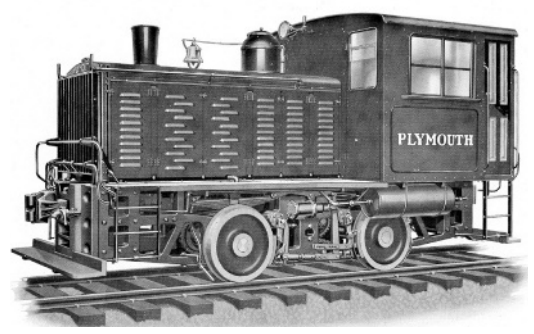

Figura 7. Dibujo de la locomotora. La familia Viosca, de La Paz, construye un ferrocarril y vía de 762 mm de ancho antes del año 1875. En 1899 tenía una locomotora de vapor y unos 40 carros para cargar la sal. En 1908, el control de la operación fue transferida a una empresa inglesa, la Compañía Salinera del Pacífico, y después de unos años de operación esporádica mejoró la recolección de sal con un equipo nuevo, incluyendo la primera de dos locomotoras de tipo Plymouth en el año 1929".

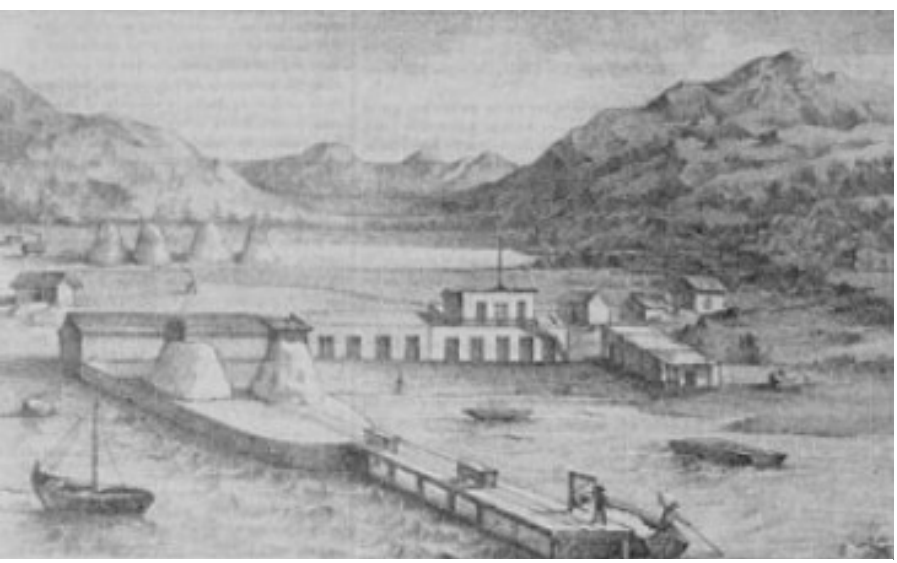
Fuente: Ferrocarriles Salineros de BCS Isla El Car- aprecia al frente del dibujo los montículos de sal en el muelle y men. Tercera de cinco partes de la exposición pre- otros montículos de sal al fondo en la laguna junto con construcsentada en el II Congreso Internacional de Antro- ciones de los almacenes, edificios de la compañía y viviendas pología de la Sal efectuado en Cabo San Lucas, obreras. Fuente: Fotos Baja California, Loreto Antiguo. California del Sur, 2017.

Alrededor de 1945 la compañía opera bajo el nombre de Pacific Salinas, S.A. y obtiene una concesión para explotar la sal durante las décadas siguientes. A mitad del siglo XX la mayoría de los socios de esta empresa eran españoles, entre ellos Cayetano Blanco Vigil, y Alonso de la Florida. Este grupo a su vez era dueño de varias salinas y tenían de Gerente General a Ricardo Sánchez. Posteriormente la salinera pasa a formar parte del conglomerado de empresas del grupo Monterrey Celulosa y Derivados, S. A. de C. V. (CYDSA) que explota la sal de la Isla del Carmen hasta 1983.

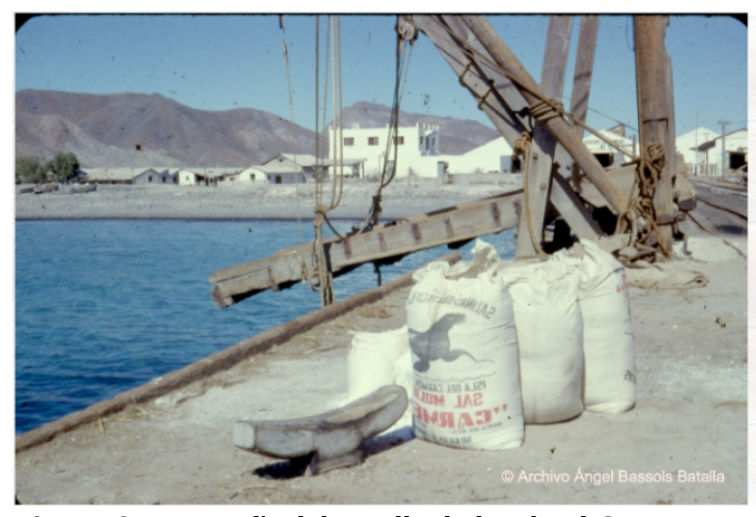

Figura 9. Fotografía del muelle de la Isla El Carmen en funcionamiento tomada por el geógrafo Ángel Bassols Batalla antes de la década de 1980. Fuente: Archivo fotográfico Ángel Bassols Batalla. COLEF.

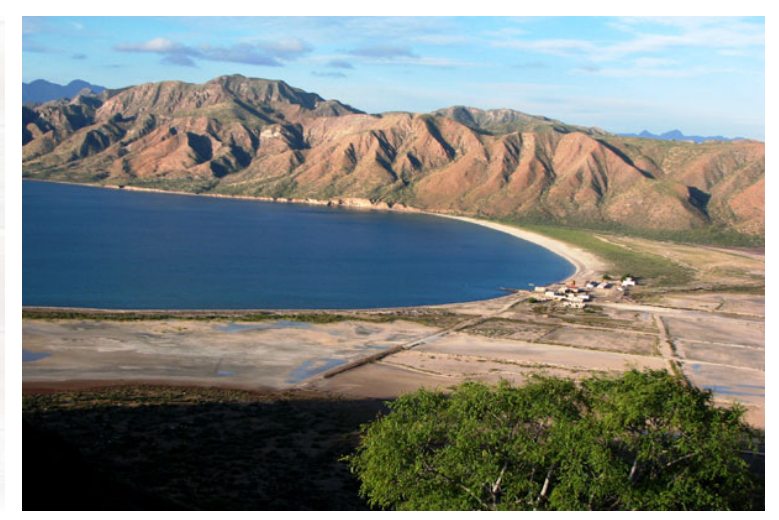

Figura 10. Fotografía de la Bahía y la salina de la Isla El Carmen.

Fuente: www.navegantecalifornio.wordpress.com

La Historia de la Isla El Carmen, como lo hemos visto gira principalmente en torno a su depósito de sal ubicado al Norte de la Isla, en la zona denominada Bahía Salinas, área donde actualmente se encuentran ubicadas la gran mayoría de las construcciones existentes, en donde se edificaron casas habitación para el personal de la salinera, además de una escuela, cementerio, canchas deportivas, talleres de trabajo, almacenes, depósitos de combustibles y aceites, así como también 
una iglesia. La actividad salinera concluye sus operaciones en 1984 quedando sus construcciones e infraestructura industrial abandonadas.

\section{Los vestigios industriales del pueblo salinero}

En 1984 la salinera cierra, pero las ruinas de las edificaciones dan la idea de lo que fue el pueblo original de la Isla. Todavía permanece el área donde se construyeron casas habitación para el personal de la salina, una escuela, iglesia, cementerio, canchas deportivas, talleres de trabajo, almacenes, depósitos de combustibles y un banco, donde en el interior de las ruinas todavía se conservan archivos.

También se encuentra la capilla de Nuestra Señora del Carmen conservada por las esposas de los dueños de la concesión. Ésta se encuentra en perfecto estado. Existen también cuatro casas bien cuidadas y confortables frecuentadas por cazadores que visitan la isla.

Una versión dice que la Isla del Carmen fue abandonada después de que se abrieron las Salinas de Guerrero Negro, cuya producción resulta mucho mayor, y especialmente su ubicación en el área del Océano Pacífico representó un gran ahorro en distancia y en costos de transportación para los clientes. Ir hasta la isla El Carmen implicaba rodear desde el Océano Pacífico todo el Estado de Baja California Sur, siendo el principal cliente de la sal mexicana Japón, les resultó más conveniente comprar la sal en Guerrero Negro, donde incluso se volvieron accionistas de la Compañía de sal. En la actualidad Mitsubishi Corporation cuenta con el 49\% de las acciones de la salinera, y el $51 \%$ pertenecen al gobierno Federal.

La realidad es que ante el éxito de la salina de Guerrero Negro, Salinas del Pacífico, en Isla El Carmen, simplemente deja de operar.

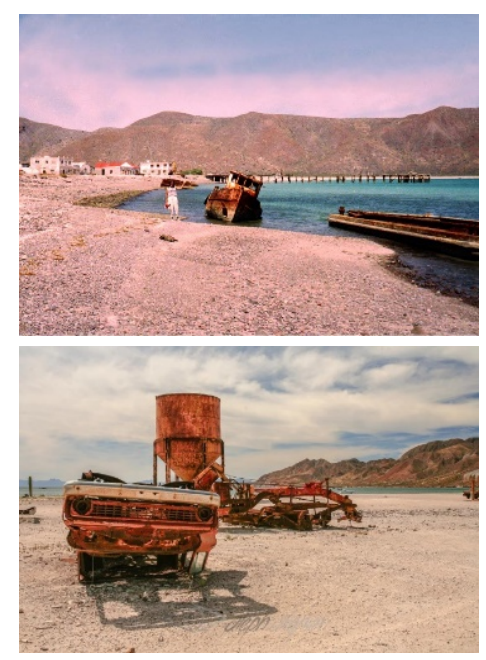

Figura 11. Fotografías de las ruinas industriales. Fuente: Del Carmen Island por Estela Davis. Fotografía de Aleph Alighieri.

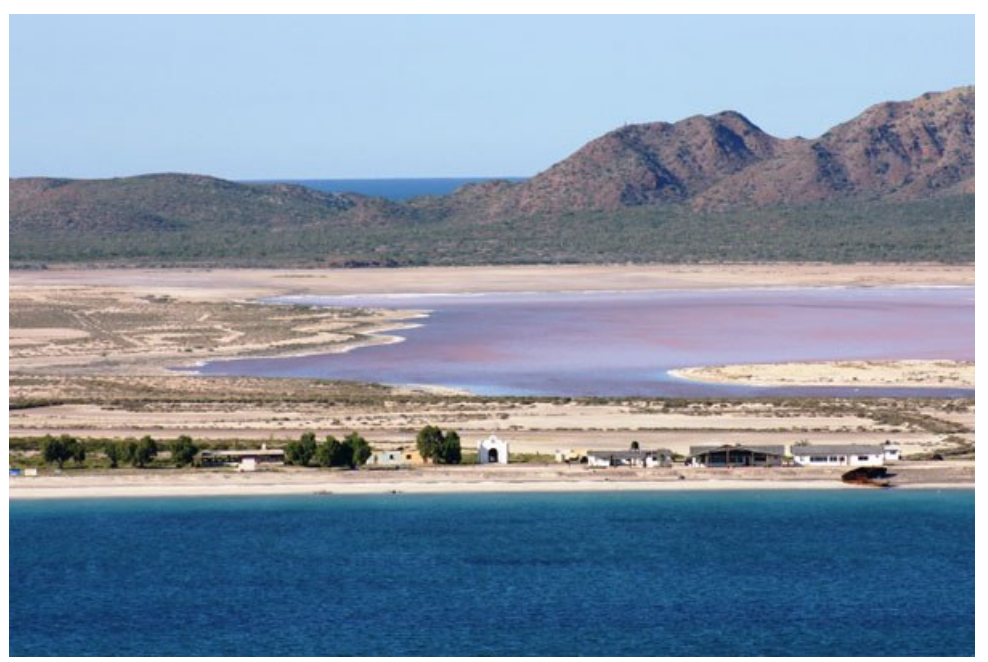

Figura 12. Fotografía de las construcciones la bahía y la laguna de sal. Fuente: El Carmen, Baja California Sur - la isla de la sal en el Mar de Cortés. Casimiro Gardea Orozco 2015.

Fotografía. http://www.mexicohunts.com/

La estructura urbana de la salina corresponde a una ordenación de una pequeña colonia industrial implementada por las compañías en diferentes etapas, la distribución de las construcciones se establece a lo largo de un eje paralelo a la playa de la bahía, el ferrocarril atravesó de forma trasversal el núcleo urbano transportando la sal desde la laguna hasta el muelle. En la parte central de la intersección se localizan los equipamientos industriales junto a la empacadora de sal, bodegas y almacenes. En la parte derecha se encuentran los edificios administrativos, el banco y viviendas obreras, en la parte izquierda se encuentran la escuela, la iglesia y otro conjunto de viviendas. 


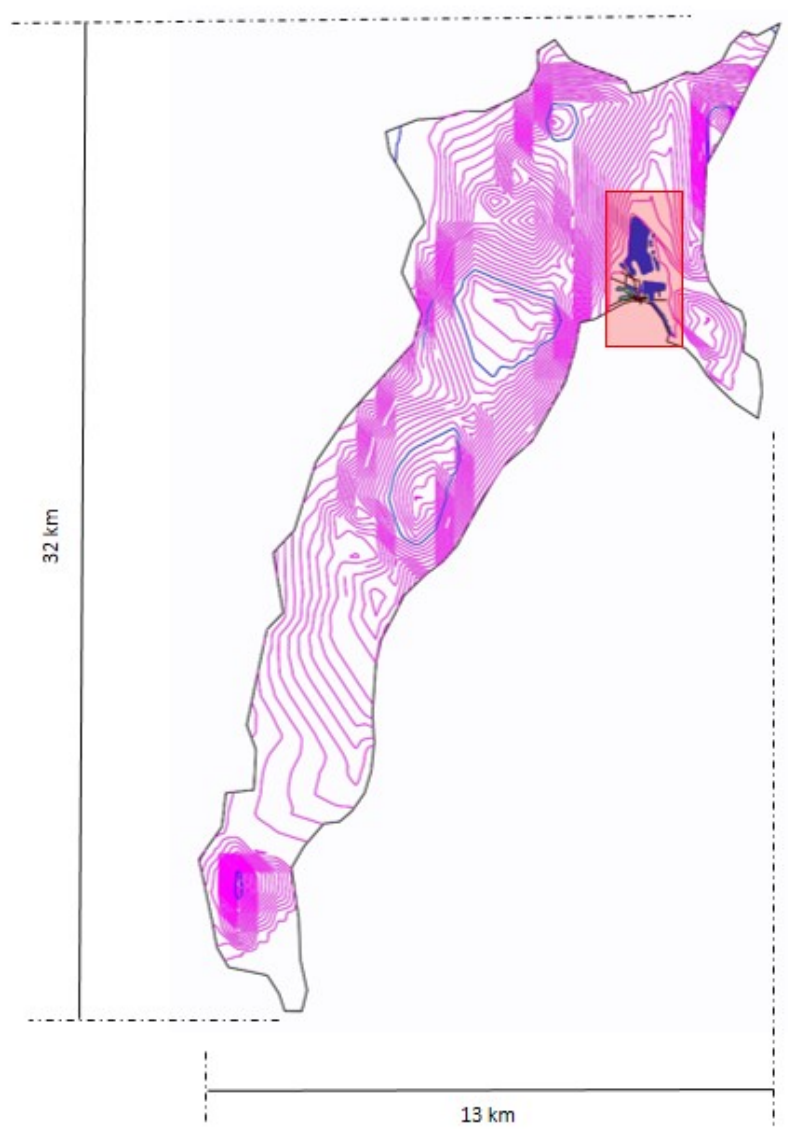

Figura 13. Plano topográfico de la Isla El Carmen en Baja California Sur y localización de la salina.

Fuente: Elaboración propia.

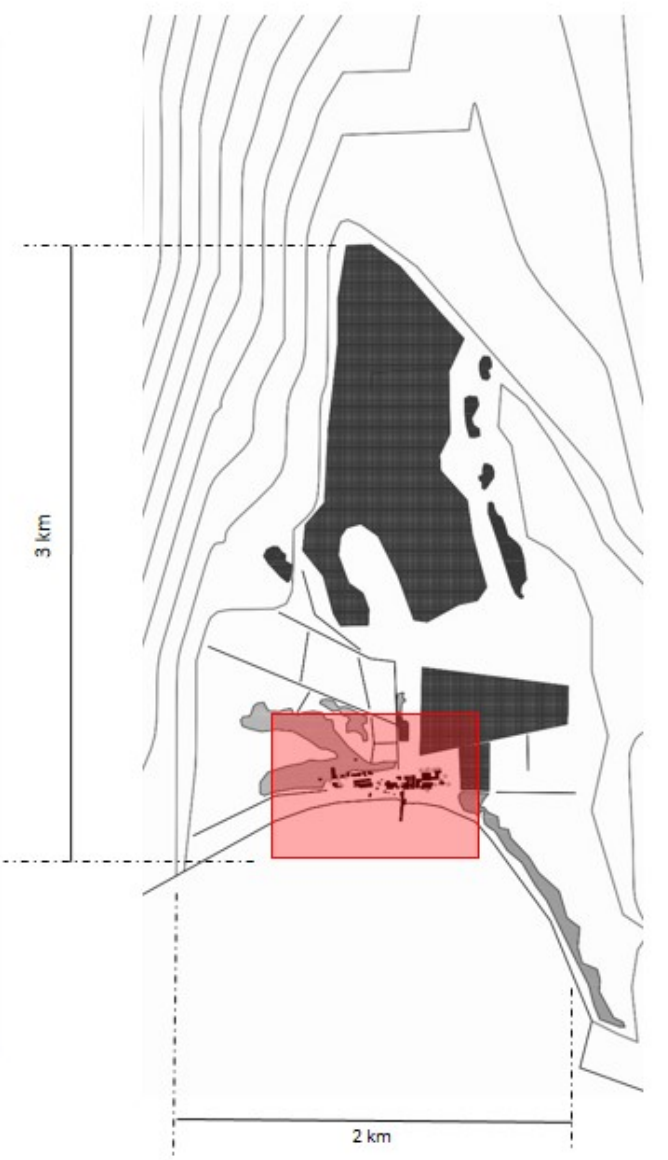

Figura 14. Acercamiento de la bahía donde se encuentra los restos de la población de la salina y la laguna. Fuente: Elaboración propia
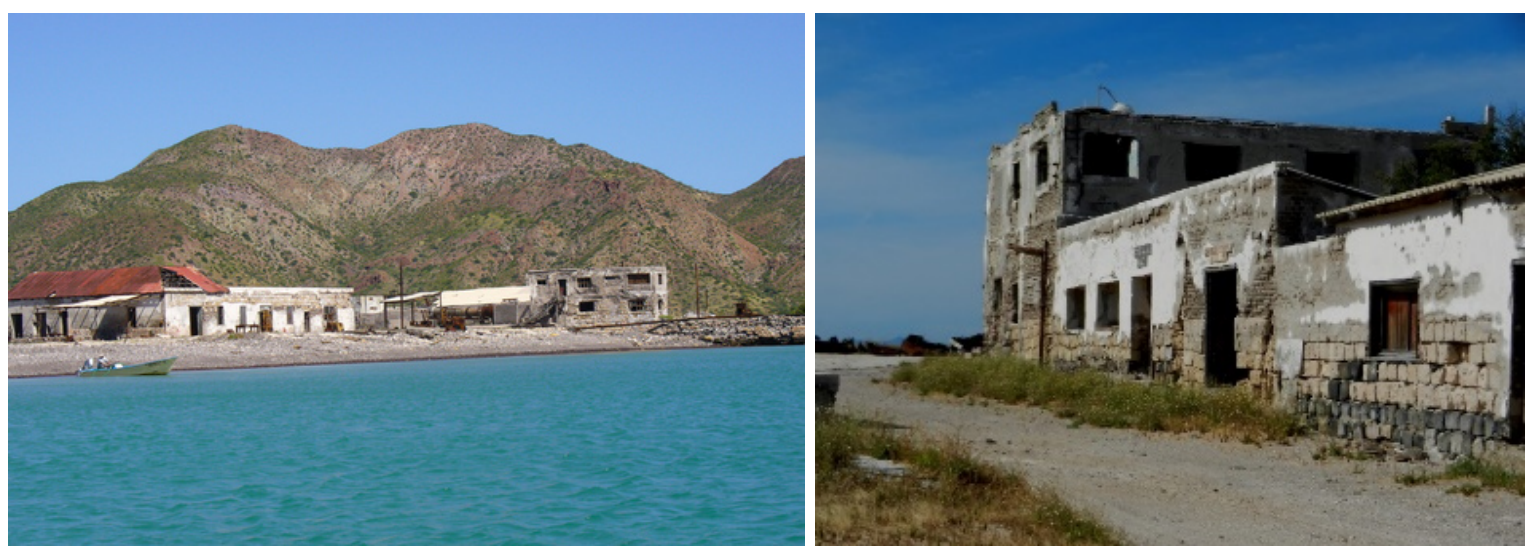

Figura 15. Fotografías de las ruinas de la empacadora de sal Fuente: Fotografía de José Sao obtenida de El Babel. El Carmen la Isla de sal en el Mar de Cortés de Benjamín Arredondo 2011.
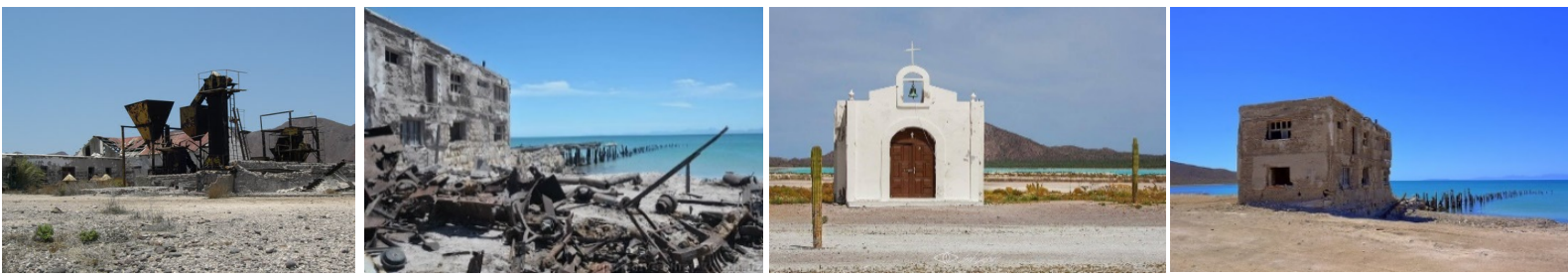

Figura 16. Fotografías de las ruinas actuales en la isla El Carmen y esquemas de análisis de su estructura urbana. Se ubican los edificios importantes y las construcciones existentes del poblado. Fuente: Fotografías de J. Davis y Geoffrey W. Schultz, 2012. 


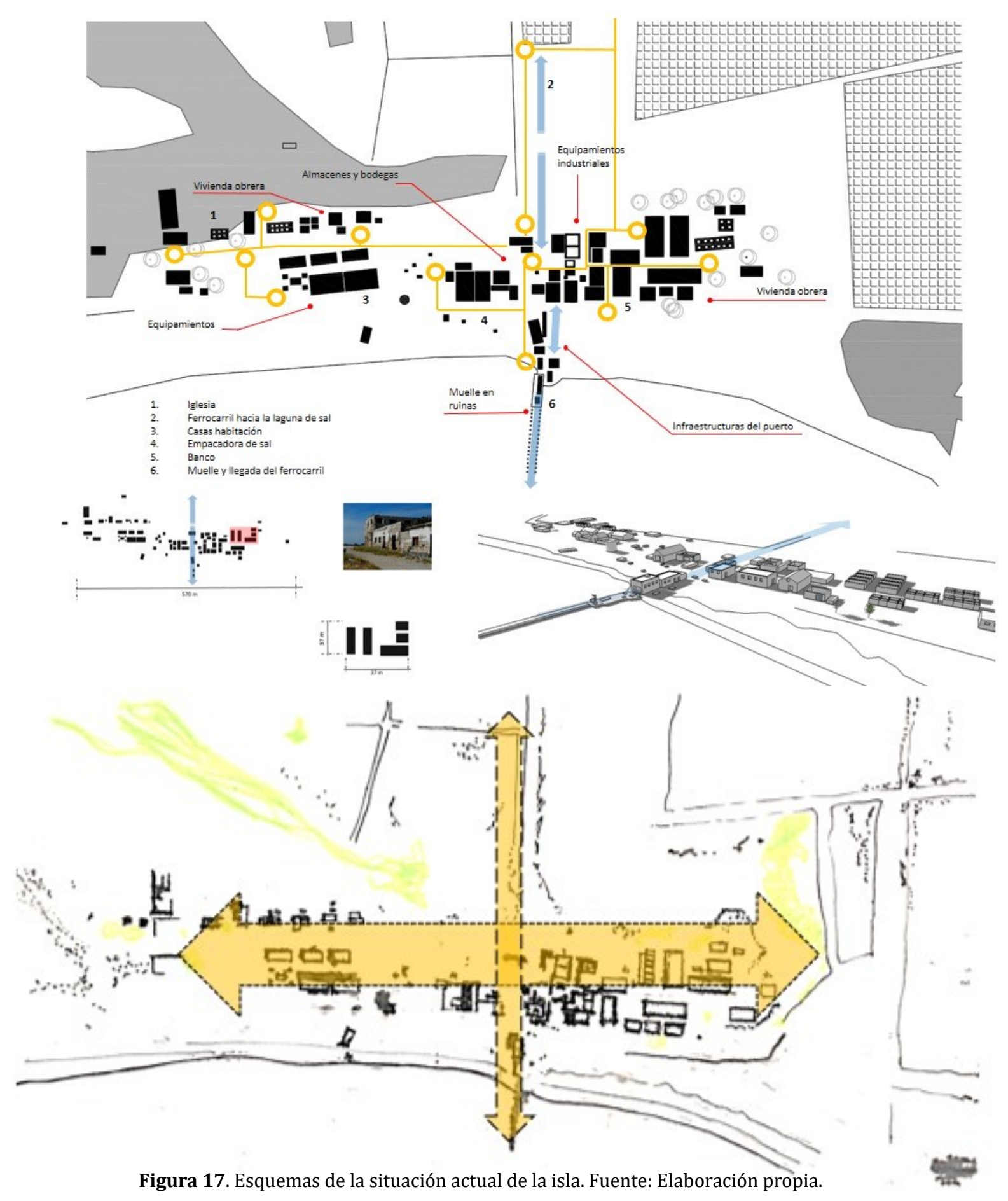

Se puede apreciar una cantidad de ruinas importante en la salina y actualmente se le puede considerar un pueblo fantasma ya que no hay residentes en la isla. Es importante hacer el reconocimiento de esta colonia industrial que se encuentra perdida en el mar de cortés, porque esta salina fue parte fundamental y de enorme relevancia en el desarrollo económico de Baja California Sur a finales del siglo XIX y principios del XX. El patrimonio industrial ha tenido un interés mundial en las últimas décadas y son muchos los ejemplos de la industrialización que comienzan a convertirse en territorios museos ${ }^{2}$.

\footnotetext{
2 Se recomienda ver "La herencia industrial y cultural en el paisaje: Patrimonio Industrial, Paisaje y Territorios Inteligentes”. De Miguel Ángel Álvarez Areces.
} 


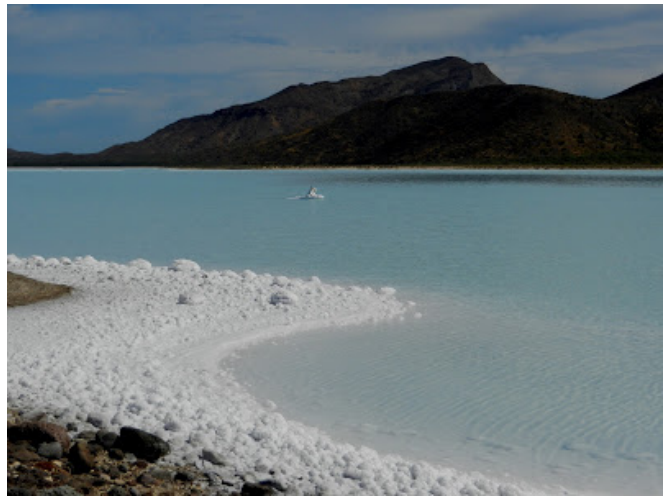

Figura 18. Fotografía de la laguna natural de sal en la Isla El Carmen. Fuente: Pacific Coast Baja Bound. Ghost Town at Bahia Salinas. 2013.

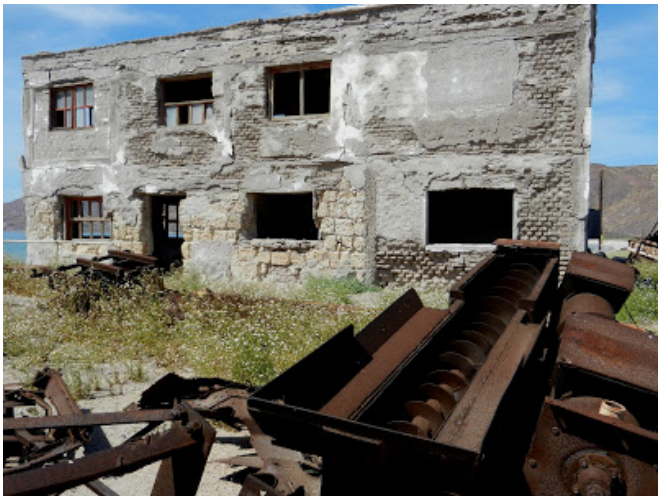

Figura 19. Fotografía de edificio en ruinas en la salina de Isla El Carmen. Fuente: Pacific CoastBaja Bound. Ghost Town at Bahia Salinas. 2013.

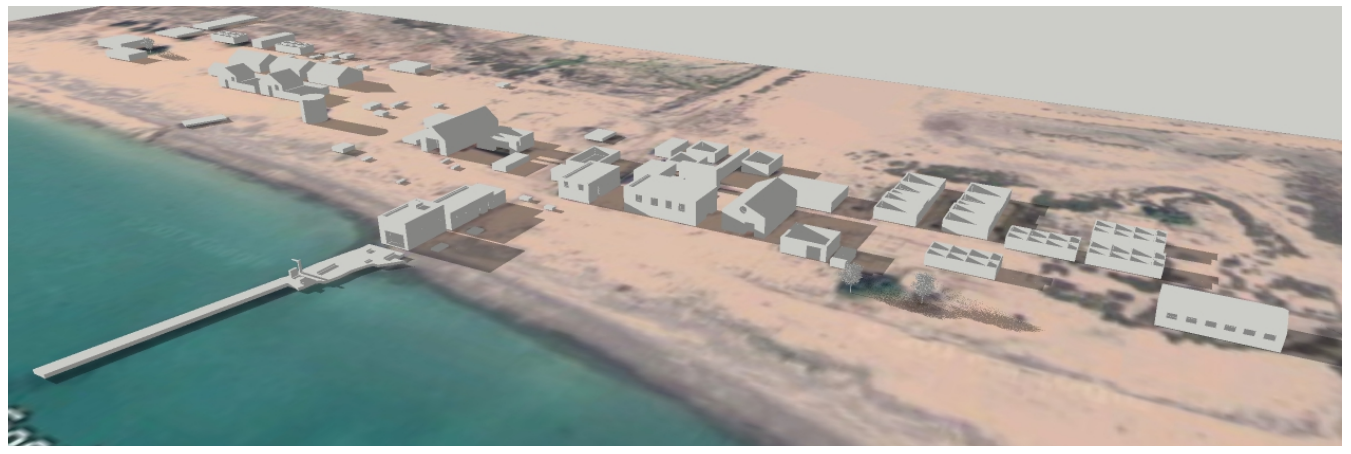

Figura 20. Esquema volumétrico de la estructura urbana en ruinas de la salina de la Isla El Carmen. Fuente: Elaboración propia.

\section{Apreciación final: Un museo de sitio salinero}

La zona donde se encuentra situada la salina de El Carmen es un área natural protegida, indicativo de la riqueza paisajista de este territorio. Un museo de sitio de la sal podría garantizar la conservación de las ruinas industriales, la arquitectura y la identidad de esas huellas de la producción salinera en la península de Baja California, que fácilmente podrían complementar esa idea de preservación de los elementos que integran este extraordinario territorio. Se es consciente que una reactivación económica en la isla es muy difícil de lograr, pero la isla se ha convertido en la actualidad en una estancia no tan conocida de patrimonio industrial visitada por turistas norteamericanos que encuentran los vestigios salineros de una company town en ruinas en sus recorridos por el mar de Cortés, una alternativa viable para conservar y catalogar las ruinas industriales.

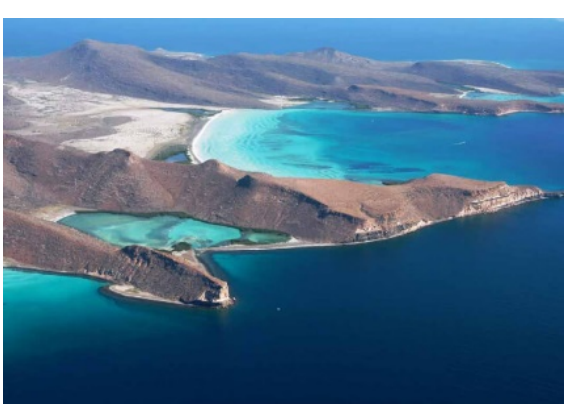

Figura 21. Foto del parque natural marino de Loreto donde la isla El Carmen funge como eje central de las demás islas. Fuente: Cucapa, experiencias en Baja.

Figura 22. Mapa del parque nacional Bahía de Loreto y su perímetro de conservación natural.

Fuente: http://

bahiadeloreto.conanp.gob.mx/
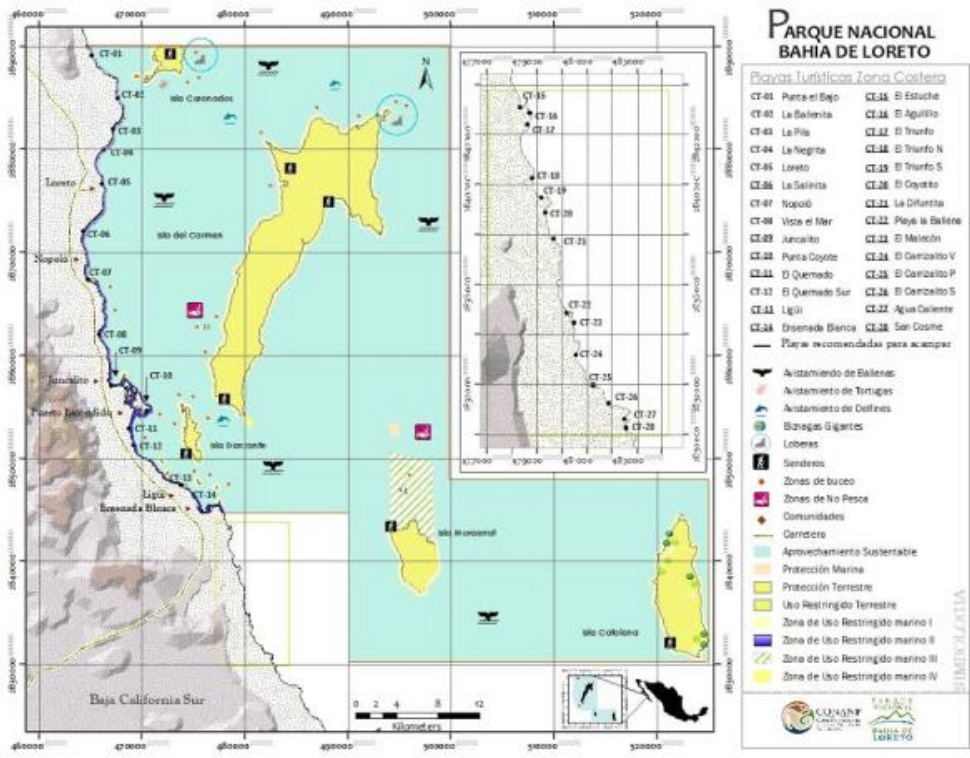


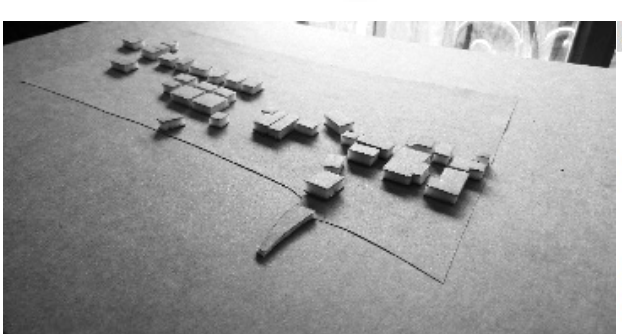

Figura 23. Maqueta de la colonia industrial salinera: Fuente: Elaboración propia.

Tomando de referencia el contexto europeo y en específico el patrimonio salinero español, encontramos numerosos ejemplos donde se han desarrollado propuestas de intervenciones para reactivar ruinas salineras, un ejemplo importante y que guarda similitudes (hasta en el nombre) con el caso bajacaliforniano, se encuentra en las Islas Canarias, en la isla Fuerteventura que cuenta con el Museo de Sal y las Salinas del Carmen en el municipio de Antigua. El recinto que funciona como museo se ubica sobre el espacio natural que ocuparon las viejas salinas que se construyeron en el siglo XVIII. Sobre las antiguas salinas se levantaron las actuales aproximadamente en 1910, respondiendo al modelo de salina nueva de barro con forro de piedra, aunque manteniendo el
Análisis territorial de una sección de interés patrimonial en la península de Baja California.

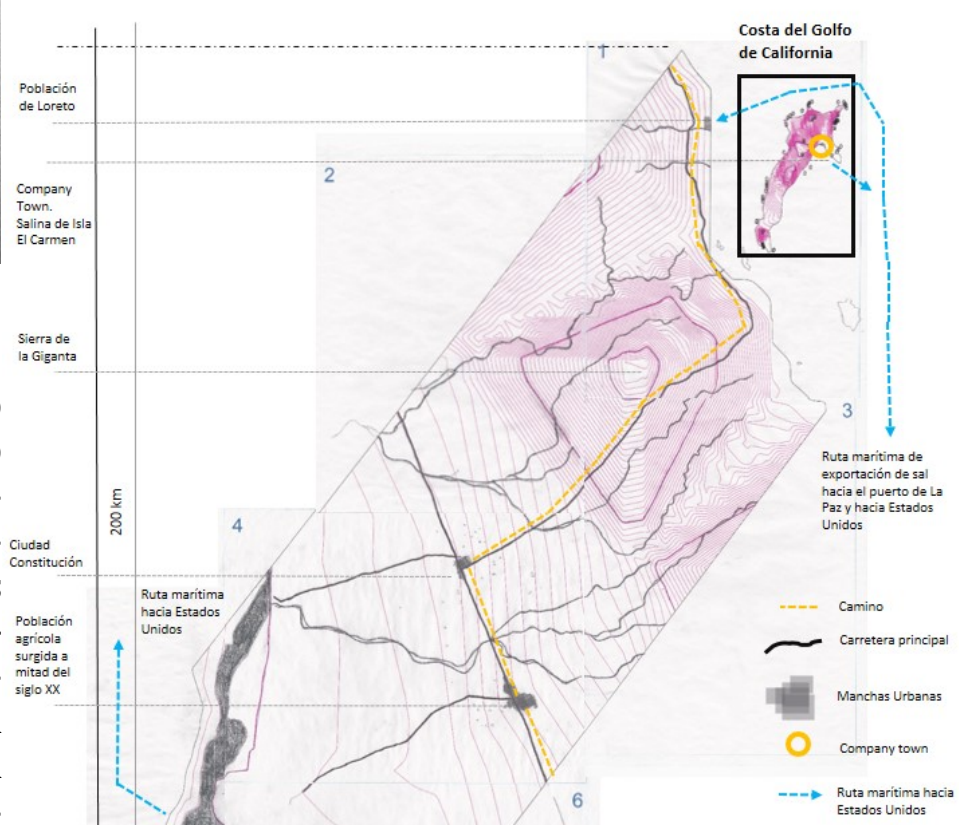

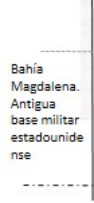

Figura 24. Esquema de un espacio territorial en el sur de la península de Baja California, donde se localiza la Isla El Carmen en el Mar de Cortés. Lo que se intenta representar en este dibujo es la dimensión de un espacio de gran riqueza natural y paisajística. Ubicando las ruinas de la company town salinera. Fuente: Elaboración propia.

tajo sencillo de las primeras salinas. A través de programas de rehabilitación en la actualidad, en las salinas se han resaltado los elementos patrimoniales y se han construido el museo de sal y contenidos museísticos de interpretación en el territorio. También se han creado recorridos por la salina donde se aprecian las características arquitectónicas, los diferentes elementos que la estructuran, y el proceso de producción de la sal (Florido, 2012).

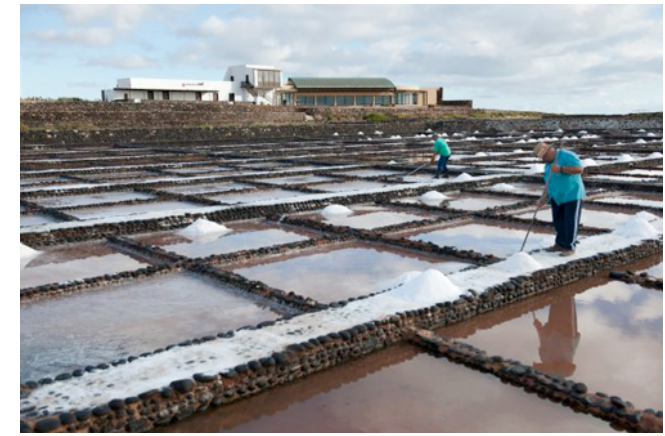

Figura 25. Museo de la sal en Fuerteventura. Fuente: http://blog.hotelesglobales.com/

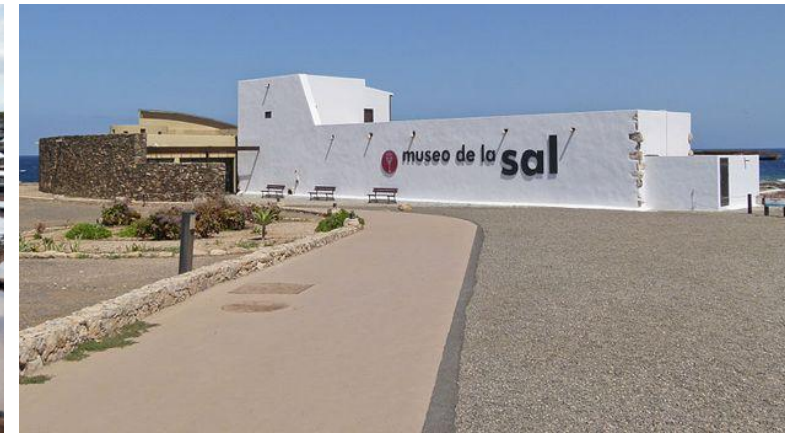

Figura 26. Edificio del Museo de Sal. Fuente: http://blog.hotelesglobales.com/

Las salinas de Fuerteventura en España son de gran interés etnográfico, arquitectónico, natural, cultural y paisajístico, el proyecto de intervención ha sabido realzar su identidad con una formidable obra de rehabilitación, constituyendo una oferta turística importante. Creemos que el caso español podría ayudar a visualizar una propuesta en la salina de El Carmen en el Golfo de 
California. Esta pequeña investigación pretende hacer una recopilación de información histórica relevante para indagar en el funcionamiento de la colonia salinera con el objetivo de resaltar su patrimonio industrial.

Se percibe un territorio rico en recursos por valorizar que cuenta con un patrimonio industrial en riesgo por el abandono que presenta, pero de enorme valor cultural y arquitectónico ya que representa un modelo urbano de enclave industrial dedicado a la extracción y exportación de sal que aparece a finales del siglo XIX. Hoy en día solo queda un pueblo fantasma en la isla y esas huellas de la industrialización que hay que poner en valor.

\section{Referencias}

Aitchison, S. (2010). The Desert Islands of Mexico's Sea of Cortez. University of Arizona Press, p.96.

Álvarez A., M. Á. (2010). La herencia industrial y cultural en el paisaje: Patrimonio Industrial, Paisaje y Territorios Inteligentes". Labor \& Engenho, 4 (1) pp.78-100.

Busto, K. (2015). Historical Archaeology of Baja California Sur. Pacific Coast Archaeological Society Quarterly, 51(3\&4), pp. 91-116.

Castro, E. \& Cariño, M. (2002). Estudio de los contratos para la explotación de sal durante el porfiriato en Sudcalifornia. Revista Clío, Nueva Época, 1 (38).

Florido, A. (2012). Los inventarios del patrimonio histórico industrial: Gran Canaria y Fuerteventura. In: XX Coloquio Historia Canaria-americana. Cabildo Insular de Gran Canaria. Las Palmas [Gran Canaria] España.

Ganster, P. (2007). Loreto: The future of the first capital of the Californias. SCERP and IRSC publications. San Diego State University. Institute for Regional Studies of the Californias. San Diego State University Press.

Pacheco, C. \& Sànchez, M. (1997). La controversia acerca de la política de colonización en Baja California. Universidad Autónoma de Baja California UABC, Mexicali Baja California.

Southworth, J. R. (1899). Baja California Ilustrada. Publicación gubernamental. La Paz, B.C. Sur, México: Gobierno del Estado de Baja California Sur.

Trejo, D. \& González, E. (2002). Historia general de Baja California Sur: La economía regional. Editores Plaza y Valdés. 